\title{
Predictive Score of Nodal Involvement in Endometrial Cancer Patients: A Large, Multicenter Series
}

\author{
Vito Andrea Capozzi, $\mathrm{MD}^{1}$ (D) , Giulio Sozzi, $\mathrm{MD}^{2}$, Andrea Rosati, $\mathrm{MD}^{3}$, Stefano Restaino, $\mathrm{MD}^{3}$, \\ Giulia Gambino, $\mathrm{MD}^{1}$, Alessandra Cianciolo, $\mathrm{MD}^{1}$, Marcello Ceccaroni, $\mathrm{MD}^{4}$, Stefano Uccella, $\mathrm{MD}^{5}$, \\ Franchi Massimo, $\mathbf{P h D}^{5}$, Vito Chiantera, $\mathbf{P h D}^{2}$, Giovanni Scambia, $\mathrm{PhD}^{3}$, Francesco Fanfani, $\mathrm{PhD}^{3}$, and \\ Roberto Berretta, $\mathrm{MD}^{1}$
}

${ }^{1}$ Department of Medicine and Surgery, University of Parma, Parma, Italy; ${ }^{2}$ Department of Gynecologic Oncology, University of Palermo, Palermo, Italy; ${ }^{3}$ Department of Woman and Child Health and Public Health, Woman Health Area, Fondazione Policlinico Universitario A. Gemelli IRCCS, Rome, Italy; ${ }^{4}$ Department of Obstetrics and Gynecology, Gynecologic Oncology and Minimally-Invasive Pelvic Surgery, International School of Surgical Anatomy, IRCCS "Sacro Cuore - Don Calabria" Hospital, Negrar, Verona, Italy; ${ }^{5}$ Department of Obstetrics and Gynecology, University Hospital of Verona, University of Verona, Verona, Italy

1. The preoperative predictive score is a good diagnostic test to identify patients at low risk of nodal metastases.

2. The risk of nodal metastasis is extremely low in the case of a negative score.

3. The application of the present score associated with the SLN algorithm could avoid unnecessary lymphadenectomies (https://doi.org/10.1245/s10434-02111083-x).
Supplementary Information The online version contains supplementary material available at https://doi.org/10.1245/s10434021-11176-7.

Publisher's Note Springer Nature remains neutral with regard to jurisdictional claims in published maps and institutional affiliations.

(C) Society of Surgical Oncology 2021

V. A. Capozzi, MD

e-mail: vitoandrea.capozzi@ @studenti.unipr.it 